\title{
Simplified Overflow Analysis of an Optical Burst Switch with Fibre Delay Lines
}

\author{
Conor McArdle, Daniele Tafani, Liam P. Barry, Anthony Holohan and Thomas Curran \\ Research Institute for Networks \& Communications Engineering, School of Electronic Engineering, \\ Dublin City University, Ireland \\ mcardlec@eeng.dcu.ie
}

\begin{abstract}
We develop an approximate analytic model of an Optical Burst Switch with share-per-node fibre delay lines and tuneable wavelength converters by employing Equivalent Random Theory, an approach from circuit-switching analysis. Our model is formulated in terms of virtual traffic flows within the switch from which we derive expressions for burst blocking probability, fibre delay line occupancy and mean delay, which we then resolve numerically. Emphasis is on simplicity of the model to achieve good numerical efficiency so that the method can be useful for formulating dimensioning problems for largescale networks. Solution values from the analysis are compared with discrete-event simulation results.
\end{abstract}

\section{INTRODUCTION}

The realisation of optical network technologies may hold the key to delivering the bandwidth demanded by the next generation of Internet applications and broadband services. The advent of optical network architectures based on wavelength division multiplexing (WDM) has gone some way to unleashing the available capacity of already deployed optical fibre in core networks; however, current network cores, employing circuit-switched overlays, are not ideally suited to efficient transport of bursty Internet traffic. Optical packet switching (OPS) [1] is regarded as the long-term solution for optical transport of Internet traffic but is not yet commercially viable. Optical burst switching (OBS) [2], [3] is a recently proposed alternative which is considered to be a strong contender as a medium-term solution to the challenge of realising available optical capacity. OBS offers a very high bit-rate transport service that is potentially bandwidth-efficient, cost-effective in terms of network infrastructure, and is designed specifically with bursty traffic in mind.

In OBS, data packets are aggregated into large bursts before transmission over the network. The transmission of the burst is then preceded by a short burst header packet (BHP), on a dedicated control channel, which requests a reservation of an optically transparent path through switches across the network to the destination. Burst transmission is delayed sufficiently at the network edge so that the BHP arrives in time to configure the switches along the path before arrival of the burst at each switch. This burst switching scheme has the desirable features of reducing packet processing overhead and avoiding the need for optical-electronic-optical conversions at switching

This material is based on research supported by Science Foundation Ireland (SFI) under the Research Frontiers Programme Grant No. [08/RFP/CMS1402]. nodes in the transport path. With optically transparent paths, there are no queuing facilities at switching nodes, or limited queuing facilities by way of fibre delay lines (FDLs). As a result, coincident bursts at the input ports of a switch must contend for free wavelength channels on output ports. This contention gives rise to burst-blocking (loss of data bursts) in the network, which is the dominant factor determining OBS network performance.

In recent years, considerable research effort has been focused on developing efficient OBS burst aggregation methods [4]-[6], optical path reservation protocols [7]-[9], and burst contention-resolution schemes [10]-[13]. Although OBS technologies are stabilising to the extent that test-beds have been built [14], [15] and it seems likely that OBS may be deployed in the medium-term, there is still a need to resolve pertinent network design, dimensioning and cost-optimisation challenges to enable deployment of OBS networks. To this end, efficient analysis methods for OBS node and network performance are desirable and considerable attention is now focused there [16]. In particular, the analysis of wavelength conversion schemes and FDLs, as two of the main contentionresolution components of the switch, is receiving attention.

The addition of wavelength converters to the switch reduces contention at output ports by enabling a burst arriving on one wavelength channel to be directed to a different wavelength channel at the output. In performance evaluation studies, there may be assumed restrictions on the number of available wavelength converters [17] and on the sharing strategy, for example, a pool of converters may be shared amongst all output ports at the switch or each output port may have a dedicated pool. Additionally, there may be restrictions on the range of conversion between one wavelength and another, due to limiting physical properties of the conversion devices [18].

The addition of FDLs to the switch can also achieve a substantial reduction in burst loss (by orders of magnitude [19]) by selectively delaying bursts in order to reduce contention for outgoing wavelengths. Our focus in this paper is on the analysis of burst loss and delay in OBS nodes with FDLs, where wavelength conversion is assumed to be unrestricted. We develop a relatively simple approximate model of FDL performance by applying classical circuit-switching analysis. Our overall aim is to produce a simple model, of good numerical efficiency, that may be extended to modelling and dimensioning of large networks of OBS switches. 
The remainder of the paper is organised as follows. Section II reviews related work on FDL performance analysis and briefly outlines the literature on the analysis method of interest in this paper; Equivalent Random Theory (ERT). Section III introduces the OBS node architecture under study. In Section IV we develop an overflow model representation of blocking at switch output ports and in FDLs. The analysis method for resolving this flow model is presented in Section V. Numerical methods are developed for approximating the probability of burst blocking and also mean delay in the switch. Section VI presents the solution values from the analysis, with comparison to discrete-event simulation results. Our conclusions and a note on future work are given in Section VII.

\section{RELATED WORK}

There are several existing approaches to performance evaluation of OBS nodes with buffering functionality implemented with FDLs. In [20], Yoo et al. provide a framework for a QoS class scheme in which they model the OBS node with FDLs as an $M / M / k / k$ queue (for classless bursts) and as an $M / M / k / D$ queue (for prioritised bursts). They assume unrestricted wavelength conversion in the switch and FDLs are organised in a feed-forward configuration. In [21], Fan et al. consider a similar switch architecture but generalise the approach. They model the FDL buffer as an $M / M / k / D$ queue and provide bounds for the loss probabilities for classless and prioritised bursts. In [22], Callegati presents a framework for evaluating the blocking probability for asynchronous variable length bursts and models a single FDL as a queue with balking. Lu \& Mark [19] model overall system behaviour as a multi-dimensional continuous-time Markov chain and also develop an asymptotic approximation model of FDLs considering separately the cases of short FDLs and long FDLs. In the former case, the FDL is modelled as an $M / M / k$ queue with balking while in the latter case, the FDL is modelled as an $M / M / k / k+m$ queue. An exact Markov chain analysis is also provided by Rogiest et al. [23] and an analysis for correlated arrivals is considered in [24]. Gauger [25] investigates the influence of the combination of wavelength converters and FDL buffers. The performance of several scenarios of feedback and feed-forward schemes are evaluated as well as the performance impact of different reservation strategies.

The approach in the current paper differs in that it is based on resolving a network of relatively simple queuing systems representing virtual traffic flows within the node. We use a circuit-switching analysis method, Equivalent Random Theory (ERT), to resolve the model. Reviriego et al. [26] have applied ERT to an approximate analysis of shared wavelength converters in an OBS node. We are not aware of any previous application of ERT to the analysis of FDLs, however. In contrast to our approach, previous work has mainly focused on detailed evaluation of single queueing systems.

Equivalent Random Theory (ERT) (also known as the Wilkinson-Bretschneider Method) [27] is a moment-matching technique which was originally developed for analysing hierarchical circuit-switched networks where overflow (blocked traffic) from a primary trunk group (or set of channels), which is offered Poisson traffic, is directed to a secondary group, rather than being discarded from the system. As overflow from a group is "peaked" (the variance of the traffic intensity is greater than the mean) and not Poisson, the primary and secondary systems must be analysed in conjunction. This analysis is the basis of ERT.

An extension of the above allows blocking probability to be resolved for any peaked traffic source offered to a blocking system by employing a two moment approximation instead of requiring a full statistical description. The analysis is carried out by substituting the actual peaked traffic source with a moment-matched overflow traffic equivalent emanating from a virtual system composed of an equivalent group of $N^{*}$ channels offered traffic of intensity $A^{*}$. This gives an acceptable approximation and makes it possible to easily evaluate performance parameters of the real system (carried and overflow traffic intensity moments, call and time congestion, etc.). These methods originate from the well-known Overflow Theory developed by Kosten and Brockmeyer [27], [28].

\section{SWITCH ARCHITECTURE}

Our model focuses on the analysis of burst blocking probability and delay in an optical burst switch having $N$ wavelength channels at each of $P$ input/output ports and a shared bank of $K$ fibre delay lines (Fig. 1). The switch employs full wavelength conversion. Tuneable converters on each input channel allow a burst on any incoming channel to be switched to any available outgoing channel or to any available channel in an FDL. The number of bursts that may be carried simultaneously by an FDL is determined by the number of tuneable converters $R$ in its return port, where $R$ is in the range $1 \leq R \leq N$. The total number of wavelength channels provided by the bank of FDLs is denoted $L=K \cdot R$. Each FDL offers a constant delay time of $D_{k}$ seconds, $k \in\{1,2, \ldots, K\}$. Delay times of the FDLs are each a multiple of a base delay time $C$ so that $D_{k}=k C$.

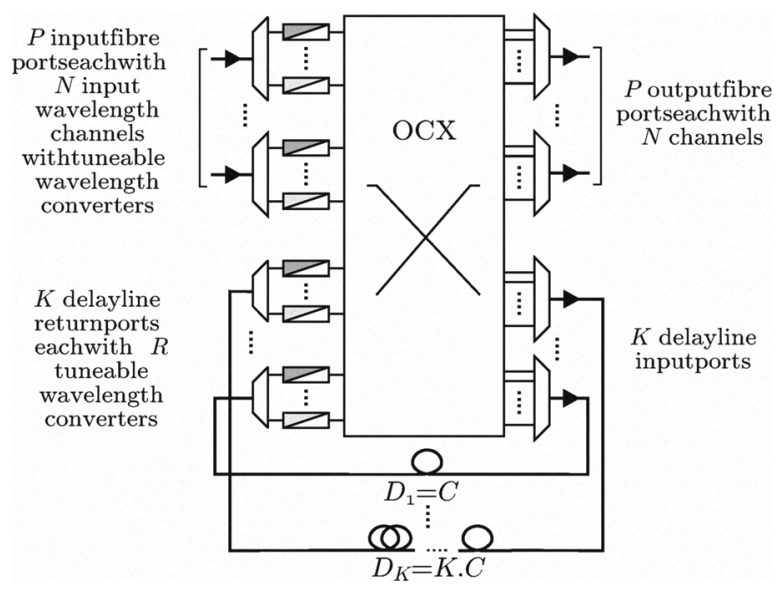

Fig. 1. Burst Switch with Tuneable Wavelength Converters and Shared FDLs 
A controller in the switch coordinates scheduling of the output port channels and FDLs. If none of the $N$ output channels of the destination output port is available for the duration of a burst arriving at a time $t$, an attempt is made to simultaneously schedule a free FDL (of delay length $D_{k}$ ) and any output channel that will become free at time $t+D_{k}$. The scheduler first attempts the procedure using FDL unit 1, offering delay $D_{1}$, and iterates in sequence through all $K$ FDLs until a feasible schedule is found. If none of the available FDL delay times can resolve the schedule, then the burst is blocked (lost).

We note that, although this architecture would allow multiple feed-back recirculations of a burst in the FDL bank, we assume only one recirculation is allowed due to signal loss constraints. This type of scheme may be realised by a tune and select (TAS) switch, as described in [29]. We next develop an analytic model which is an approximate analogue of the switch scheduling behaviour just described.

\section{OVERFLOW MODEL}

We assume that traffic arriving on input channels is Poisson in nature and that burst lengths are exponentially distributed. Traffic at output port channels is not Poisson, however, as some proportion of arriving traffic is blocked, recirculated in delay lines and subsequently carried by output channels some time after its original arrival time. Under these conditions, we may describe an output port as a $G / M / N / N$ system, a fully accessible group of $N$ channels receiving generally distributed arrivals. We wish to resolve the probability of blocking and mean burst delay at an output port, without resorting to a complex probabilistic description of the general arrival stream. We develop a simplified overflow model (Fig. 2) for this purpose.

We make the observation that the traffic which is potentially blocked by an output port, before the scheduler attempts to resolve conflicts by delaying bursts in the FDLs, may be approximated as a (virtual) overflow traffic from the $G / M / N / N$ system representing the group of output channels in a single port. This overflow is indicated in Fig. 2 as flow $\hat{\mathcal{F}}$ while the aggregation of all overflows from all output ports is denoted as $\hat{\mathcal{F}}_{T}$. We assume that these virtual overflows from different output ports are independent.

We next consider FDL 1 in the shared bank of FDLs as a group of $R$ channels that is offered all virtual overflow traffic from the output ports. We note that blocked traffic which cannot subsequently be carried by the output port, having been delayed, should not properly be considered as part of offered traffic to the FDL because the scheduler ensures that delay on an FDL is only attempted if the delayed burst may subsequently be carried by the output port. However, assuming that FDLs are longer than the mean burst length, we view traffic emanating from an FDL as competing only with other delayed bursts for output channels and so the blocking probability of delayed bursts is taken to be negligible compared to blocking arising from the original offered traffic at the output port.

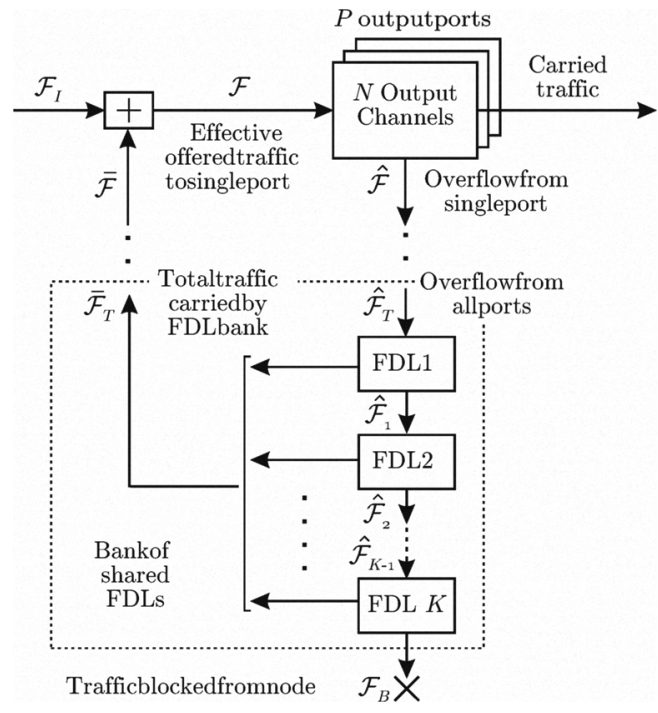

Fig. 2. Virtual Flow Model of OBS Output Ports with Shared FDLs

For FDL 1, when all $R$ channels are busy the scheduler cannot resolve a conflict using FDL 1 and instead attempts to resolve it with the delay offered by FDL 2 . Thus we view FDL 1 as a blocking system generating its own overflow traffic $\hat{\mathcal{F}}_{1}$ which in turn is offered to FDL 2, and so on down the chain of $K$ FDLs, with each FDL $k$ producing overflow $\hat{\mathcal{F}}_{k}$. Overflow $\hat{\mathcal{F}}_{K}$ from the final FDL represents the actual overflow from the node. This traffic flow, $\mathcal{F}_{B}=\hat{\mathcal{F}}_{K}$, is lost. Thus, the FDL bank is modelled as a chain of $G / M / R / R$ systems with cascading overflows, where $R$ is the number of channels in each FDL.

We assume that the offered traffic to the first FDL in the chain is composed of independent arrivals (a renewal stream). Under this assumption, in addition to an individual $G / M / R / R$ model for each FDL, we may model the combined chain of $K$ FDLs as a single $G / M / L / L$ system where $L=K \cdot R$ is the aggregate number of channels in the FDL bank. This $G / M / L / L$ model directly relates the traffic offered to the FDLs, $\hat{\mathcal{F}}_{T}$, to the carried and overflow traffics, $\overline{\mathcal{F}}_{T}$ and $\mathcal{F}_{B}$ respectively.

To complete the model, we consider the combined traffic carried by all FDLs in the bank as a traffic flow that will be offered again (notionally) to the output ports, at some time in the future. This total carried traffic flow from the FDLs is indicated as $\overline{\mathcal{F}}_{T}$ and is split into $P$ sub-streams $(\overline{\mathcal{F}})$ each being returned to a single output port. For simplicity of model formulation, we assume that all input ports receive identical arrival streams $\left(\mathcal{F}_{I}\right)$, distribute their traffic evenly to all $P$ output ports which generate identical overflow streams and then receive identical return flows from the FDL bank. Under these homogeneity assumptions, the input flow to each port, $\left(\mathcal{F}_{I}\right)$, is analogous to the offered traffic flow at an output port.

We finally identify an effective (virtual) offered traffic flow $\mathcal{F}$, at each port, that is the aggregation of the offered traffic flow $\left(\mathcal{F}_{I}\right)$, and the additional FDL carried traffic $(\overline{\mathcal{F}})$ which is 
fed back to the input of the model. Our aim is to resolve $\mathcal{F}$ approximately by resolving the first two moments of all flows in the model. We note that although we choose Poisson traffic for input port arrivals, in what follows, the model may equally admit more general offered traffic by means of specify $\mathcal{F}_{I}$ by its first two moments. A summary of the main flows in the model is as follows:

- $\mathcal{F}_{I}$ is the actual traffic flow offered to a single output port. This flow is assumed to be Poisson. The flow's mean intensity is given as $M_{I}$ and the variance as $V_{I}=M_{I}$.

- $\mathcal{F}_{O}$ is the total actual carried traffic from the node, with mean intensity $M_{O}$ and variance $V_{O}$.

- $\mathcal{F}_{B}$ is the total actual blocked (lost) traffic from the node, with mean intensity $\hat{M}_{B}$ and variance $\hat{V}_{B}$.

- $\hat{\mathcal{F}}$ is the overflow traffic from a single output port modeled as a $G / M / N / N$ system. The mean and variance of the flow's intensity are $\hat{M}$ and $\hat{V}$ respectively.

- $\hat{\mathcal{F}}_{T}$ is the aggregation of all the overflows from the $P$ output ports. This flow constitutes the overall traffic that must either be delayed and scheduled on output channels for transmission at a later time, or else blocked if there is no feasible schedule. The mean and variance of the flow's intensity are $\hat{M}_{T}$ and $\hat{V}_{T}$ respectively.

- $\overline{\mathcal{F}}_{T}$ is the carried traffic from the $G / M / L / L$ system. This flow represents the total traffic that is successfully scheduled to be delayed in the FDL bank and subsequently carried by the output ports. The mean and variance of the flow are $\bar{M}_{T}$ and $\bar{V}_{T}$ respectively.

- $\overline{\mathcal{F}}$ is the portion of the carried traffic from the $G / M / L / L$ system being offered to a single output port. The mean and variance of the flow are $\bar{M}$ and $\bar{V}$ respectively.

- $\mathcal{F}$ is the effective total offered traffic to a single output port. This consists of the actual offered traffic to the port output channels plus a portion of the traffic flow generated by previously delayed traffic from the FDL bank. Its mean intensity is $M$ and its variance $V$.

\section{Model AnAlysis}

We next analyse the model to resolve the mean and variance of the flows identified in the previous section using methods from Equivalent Random Theory. Having done this we may estimate the burst blocking probability at the node and the mean delay experienced by a burst transiting the switch.

\section{A. Resolving Blocking Probability}

We first resolve $\hat{M}$ and $\hat{V}$, the mean and the variance of the overflow traffic $\hat{\mathcal{F}}$ from a single output port, given that the offered traffic flow to the port is described by its mean and variance $M$ and $V$ respectively. We assume that this offered traffic has a "peakedness" $Z>1$, where $Z=V / M$, and apply Brockmeyer's method [27] to resolve the overflow moments.

By way of explanation of the method, we first introduce the Kosten overflow equations [28], which allow resolution of the moments of the overflow traffic from a group of $N$ channels when the offered traffic is Poisson $(Z=V / M=1)$. In this case, the mean and variance of the overflow traffic are given by

$$
\begin{aligned}
& \hat{M}=M \cdot E(M, N) \\
& \hat{V}=\hat{M}\left(1-\hat{M}+\frac{M}{N+1+\hat{M}-M}\right),
\end{aligned}
$$

where $M$ and $V$ are the mean and variance of the offered traffic and the function $E(\cdot)$ is the Erlang-B formula defined as

$$
E(A, N)=\frac{A^{N}}{N !} / \sum_{k=0}^{N} \frac{A^{k}}{k !},
$$

where $A$ is the arrival intensity of the offered traffic, in units of Erlangs, and $N$ is the group size (number of channels) [30]. The arrival intensity $A$ is given as the product of the mean arrival rate $\lambda$ and the mean channel holding time $1 / \mu$ (the mean burst length).

In the case of "peaked" offered traffic, with mean and variance $M$ and $V$, to a group of $N$ channels, the Brockmeyer method makes the following moment-matching construction which is solvable by the Kosten equations. A virtual group of size $N^{*}$ is offered virtual Poisson traffic of intensity $A^{*}$ which is presumed to produce an overflow mean and variance $M$ and $V$ (Fig. 3). When this overflow traffic is then offered to the actual group of size $N$, the combined system is equivalent to a system of $N^{*}+N$ channels offered Poisson traffic of intensity $A^{*}$. The overflow from this system, which is equivalent to the overflow from $N$ channels offered traffic $(M, V)$, can be resolved using Kosten's equations as

$$
\begin{aligned}
& \hat{M}=A^{*} \cdot E\left(A^{*}, N^{*}+N\right) \\
& \hat{V}=\hat{M}\left(1-\hat{M}+\frac{A^{*}}{N+N^{*}+1+\hat{M}-A^{*}}\right) .
\end{aligned}
$$

The problem of finding overflow traffic $\hat{M}, \hat{V}$ reduces to finding the $A^{*}$ and $N^{*}$ group whose overflow matches the actual offered traffic to the actual group of $N$ channels. That

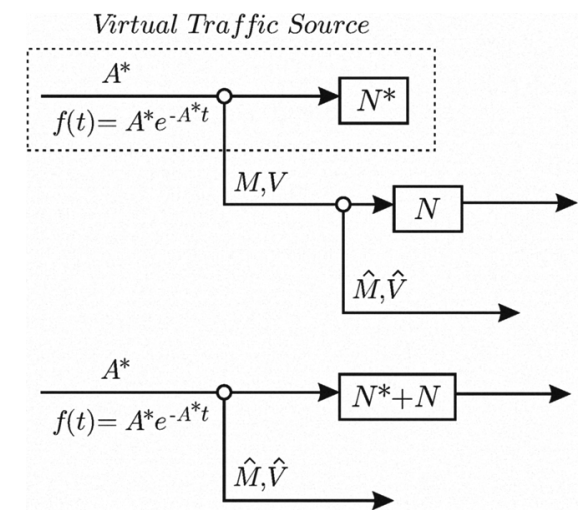

Fig. 3. Virtual Overflow System and its Equivalent for Resolving Overflow of Traffic $M, V$ Offered to $N$ Output Channels 
is, considering the $N^{*}$ group alone and given $M$ and $V$, find $A^{*}$ and $N^{*}$ such that

$$
\begin{aligned}
& M=A^{*} \cdot E\left(A^{*}, N^{*}\right) \\
& V=M\left(1-M+\frac{A^{*}}{N^{*}+1+M-A^{*}}\right)
\end{aligned}
$$

and then substitute in (4) and (5) to find $\hat{M}$ and $\hat{V}$. To solve (6) and (7), from (7) $N^{*}$ may be written in terms of $A^{*}$ and known constants $M$ and $V$ as

$$
N^{*}=A^{*}\left(\frac{M+V / M}{M+V / M-1}\right)-M-1
$$

and then, substituting for $N^{*}$ in (6), gives a function of a single variable $A^{*}$,

$$
f\left(A^{*}\right)=M-A^{*} \cdot E\left(A^{*}, N^{*}\right)=0,
$$

which may be solved for $A^{*}$ as a numerical root finding problem. We employ the Newton-Raphson method, with a good initial starting point for the algorithm given by Rapp's approximation [27]:

$$
\begin{aligned}
& A^{*} \approx V+3 Z(Z-1) \\
& N^{*} \approx \frac{A^{*}(M+Z)}{M+Z-1}-M-1 .
\end{aligned}
$$

We note that in the numerical method the values of $N^{*}$ must be allowed to take non-integer values for an exact solution to be found. The usual recurrent evaluation method for evaluating the Erlang-B formula [28] is of the form

$$
E(A, k+1)=\frac{A \cdot E(A, k)}{k+1+A \cdot E(A, k)}, \quad E(A, 0)=1 .
$$

This must be extended using Szybicky's approximation [31] which gives the blocking probability for real-valued $N, 0 \leq$ $N \leq 2$, as

$$
E_{s}(A, n) \approx \frac{(2-n) A+A^{2}}{n+2 A+A^{2}} \quad n \in \text { real interval }[0,2] .
$$

For a given positive real-valued $N=\lfloor N\rfloor+(N-\lfloor N\rfloor)$, where $N$ may be $\geq 2$, we first evaluate

$$
E(A, N-\lfloor N\rfloor)=E_{s}(A, N-\lfloor N\rfloor)
$$

and then (from (10)) form the recursion

$$
\begin{aligned}
& E(A, k+1+(N-\lfloor N\rfloor))= \\
& \frac{A \cdot E(A, k+(N-\lfloor N\rfloor))}{k+1+(N-\lfloor N\rfloor)+A \cdot E(A, k+(N-\lfloor N\rfloor))}
\end{aligned}
$$

where, for $k=0$

$$
E(A, 0+N-\lfloor N\rfloor)=E_{s}(A, N-\lfloor N\rfloor) .
$$

Iterating for $k=0,1, \ldots,\lfloor N\rfloor-1$ gives the final value of $E(A, N)$, for positive real-valued $N$.

Having calculated the mean $\hat{M}$ and variance $\hat{V}$ of the overflow traffic from an output port by the above, we now wish to calculate the mean and variance of both the overflow traffic $\hat{\mathcal{F}}$ and the carried traffic $\overline{\mathcal{F}}$ from the bank of FDLs.
The input flow offered to the bank of FDLs is taken as the aggregate of all overflow streams from the $P$ output ports in the node. Thus, the total mean intensity of the input traffic offered to the FDLs is equal to the sum of all the overflow means from each output port, namely $\hat{M}_{T}=P \cdot \hat{M}$. Assuming independence between the overflow streams from each output port, we can calculate in a similar way the total variance as $\hat{V}_{T}=P \cdot \hat{V}$.

We employ Brockmeyer's method, in the same manner as above, to calculate the mean and variance of the total overflow from the FDL bank, a group of $L$ channels offered traffic of mean and variance $\hat{M}_{T}$ and $\hat{V}_{T}$. In this case, $A^{*}$ and $N^{*}$ must satisfy the system

$$
\begin{aligned}
& \hat{M}_{T}=A^{*} \cdot E\left(A^{*}, N^{*}\right) \\
& \hat{V}_{T}=\hat{M}_{T}\left(1-\hat{M}_{T}+\frac{A^{*}}{N^{*}+1+\hat{M}_{T}-A^{*}}\right) .
\end{aligned}
$$

The mean and variance of the overflow traffic from the bank of FDLs is then given by

$$
\begin{aligned}
& \hat{M}_{B}=A^{*} \cdot E\left(A^{*}, N^{*}+L\right) \\
& \hat{V}_{B}=\hat{M}_{B}\left(1-\hat{M}_{B}+\frac{A^{*}}{N^{*}+L+1+\hat{M}_{B}-A^{*}}\right) .
\end{aligned}
$$

We may approximate the mean and variance of the carried traffic from the FDL bank simply as

$$
\begin{aligned}
\bar{M}_{T} & =\hat{M}_{T}-\hat{M}_{B} \\
\bar{V}_{T} & =\hat{V}_{T}-\hat{V}_{B} .
\end{aligned}
$$

This FDL carried traffic is returned to the output ports in our model. Assuming independence, we split the FDL carried flow $\overline{\mathcal{F}}_{T}$ into $P$ identical carried streams, each one being returned to an output port. These individual streams have the same mean intensity and variance which are respectively given by $\bar{M}=$ $\bar{M}_{T} / P$ and $\bar{V}=\bar{V}_{T} / P$. Considering the feedback connection at the input of the model, the aggregation of the actual input flow $\mathcal{F}_{I}$ and the carried FDL traffic flow $\overline{\mathcal{F}}$ gives

$$
\begin{aligned}
M & =M_{I}+\bar{M} \\
V & =V_{I}+\bar{V} .
\end{aligned}
$$

We solve this two-variable system using the Newton-Raphson method with initial values of $M$ and $V$ given by $M_{I}$ and $V_{I}$ respectively. We note, empirically, that for test cases we find the same solution more efficiently by solving independently for $M$ by Equation (17), though it is not clear if this will always find a unique solution as it is difficult to determine what closed form equation determines the relationship between $M$ and $V$, given the form of the constituent equations represented by Equations (17) and (18). Having found $M$ and $V$ we finally calculate the overall node blocking probability as the ratio of the mean intensity of the FDL overflow traffic and the mean intensity of the overall actual input traffic offered to the node, that is

$$
B=\hat{M}_{B} /\left(P \cdot M_{I}\right)
$$


We note that the above method relies on all offered traffic flows to channel groups being peaked. This is because offered traffic is matched to virtual overflow traffic from a Poisson flow, which is always peaked. However, given that we include carried flows in the model, which are less peaked than the offered traffic, we need to be careful that smooth $(Z<1)$ offered traffic does not occur in the model, which the method cannot resolve. In practice, for all reasonable numbers of output port channels $N$ and FDL channels $L$, we do not find smooth traffic occurring. We note, however, that if this were the case for a given combination of $N$ and $L$, the method could be extended quite easily by way of Nightingale's ERT method or a BPP source model [28].

\section{B. Resolving Mean Delay}

Delay in the system occurs when FDLs are employed by the scheduler to resolve contention at the output channels. To evaluate the mean delay we first resolve the mean and variance of the offered traffic to each of the $K$ FDLs. Having done so, we may then resolve the mean of the carried traffic from each FDL, $\bar{M}_{k}$. This allows us to approximate the mean occupancy of each FDL $k$ from which, given a set of FDL delay times $\left\{D_{k}\right\}$, we may approximate the mean delay in the system.

We denote the mean and variance of the overflow from FDL $k$ as $\hat{M}_{k}$ and $\hat{V}_{k}$ respectively, as per Fig. 4. As the overflow from an FDL is the offered traffic to the next FDL in the chain, the offered traffic to FDL $k$ has mean and variance $\hat{M}_{k-1}$ and $\hat{V}_{k-1}$. Having solved for $M$ and $V$, in the previous subsection, the mean and variance of the traffic offered to the first FDL in the FDL bank are given by equations (11) and (12). We may now resolve the mean and variance of the overflow from FDL $1, \hat{M}_{1}$ and $\hat{V}_{1}$ respectively, when it is offered traffic $\hat{M}_{T}, \hat{V}_{T}$ using the same Brockmeyer method from the previous subsection. Having done so, $\hat{M}_{1}, \hat{V}_{1}$ becomes offered traffic to FDL 2 and, assuming independence between flows and reapplying the Brockmeyer method, we resolve $\hat{M}_{2}, \hat{V}_{2}$ and so on down the chain of $K$ FDLs. We show the solution equations for an arbitrary FDL $k$ receiving peaked traffic $\hat{M}_{k-1}, \hat{V}_{k-1}$ and producing overflow $\hat{M}_{k}, \hat{V}_{k}$. With this solution and $\hat{M}_{0}, \hat{V}_{0}$ given by $\hat{M}_{T}, \hat{V}_{T}$ respectively, we may iterate for all $K$ FDLs in the bank.

For FDL $k$, expressions for the mean and variance of the actual overflow, in terms of the virtual group size $N_{k}^{*}$, the virtual offered intensity $A_{k}^{*}$ and the actual group size $N$ are given by the equivalent system as

$$
\begin{aligned}
& \hat{M}_{k}=A_{k}^{*} \cdot E\left(A_{k}^{*}, R+N_{k}^{*}\right) \\
& \hat{V}_{k}=\hat{M}_{k}\left(1-\hat{M}_{k}+\frac{A_{k}^{*}}{R+N_{k}^{*}+1+\hat{M}_{k}-A_{k}^{*}}\right) .
\end{aligned}
$$

$A_{k}^{*}$ and $N_{k}^{*}$ are given implicitly in terms of $\hat{M}_{k-1}$ and $\hat{V}_{k-1}$, the previously calculated mean and variance of the overflow

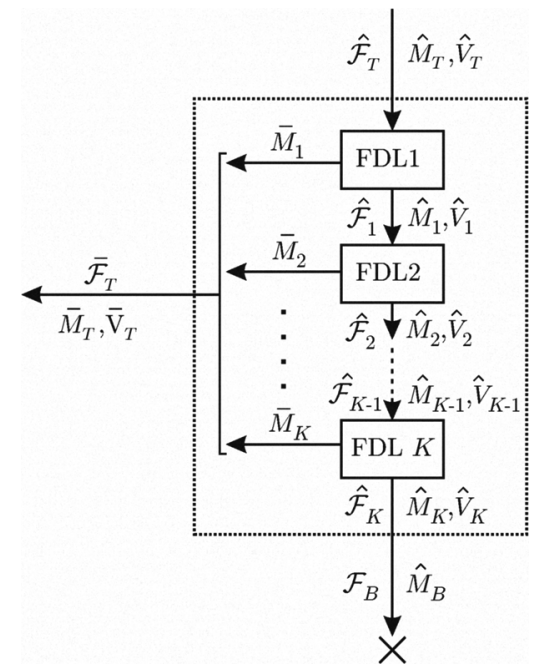

Fig. 4. Overflow System for Analysis of Individual FDLs

from the virtual source, as

$$
\begin{aligned}
& \hat{M}_{k-1}=A_{k}^{*} \cdot E\left(A_{k}^{*}, N_{k}^{*}\right) \\
& \hat{V}_{k-1}=\hat{M}_{k-1}\left(1-\hat{M}_{k-1}+\frac{A_{k}^{*}}{N_{k}^{*}+1+\hat{M}_{k-1}-A_{k}^{*}}\right) .
\end{aligned}
$$

Once $A_{k}^{*}$ and $N_{k}^{*}$ are found numerically, the mean and variance of the overflow traffic from FDL $k$ are then given by equations (20) and (21) respectively. We may now calculate the mean of the carried traffic from FDL $k$ as

$$
\bar{M}_{k}=\hat{M}_{k-1}-\hat{M}_{k} \text {. }
$$

With this solution for FDL $k$, and $\hat{M}_{0}, \hat{V}_{0}$ given by $\hat{M}_{T}, \hat{V}_{T}$, we may solve for all $k \in\{1,2, \ldots, K\}$ iteratively. The average burst delay $D$ at the output port is then given as

$$
D=\sum_{k \in\{1, \ldots, K\}} \frac{\bar{M}_{k}}{M_{O}} D_{k}
$$

where $M_{O}$ is the mean of the carried traffic from all ports, calculated as

$$
M_{O}=\left(P \cdot M_{I}\right)-\hat{M}_{B}
$$

\section{RESUlts AND ANALYSIS}

We compare analytic solutions for blocking $(B)$ and mean burst delay $(D)$ with results from discrete-event simulations of an OBS node implemented in Opnet Modeler ${ }^{\mathrm{TM}}$ [32]. The simulator models the details of the combined output channel and FDL scheduling. The channel scheduler implements Latest Available Unscheduled Channel with Void Filling (LAUC-VF) [1] on both the output channels and the FDLs. When there is no output channel available for an arriving burst, coordination of output channel and FDL scheduling is of the "PreRes" type [25]. In this scheme a schedule is sought simultaneously for 


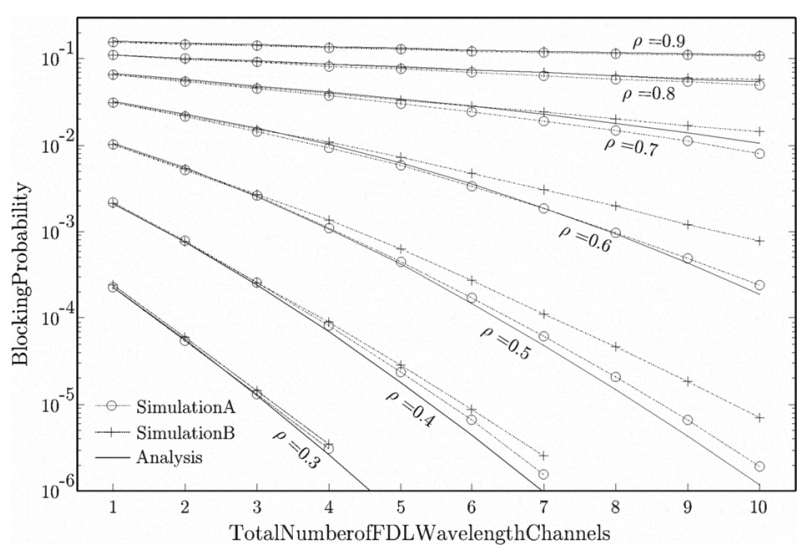

Fig. 5. Blocking Probability - Simulation and Analysis - 4 Port, 10 Output Channel Switch with two FDL Configurations (A and B)

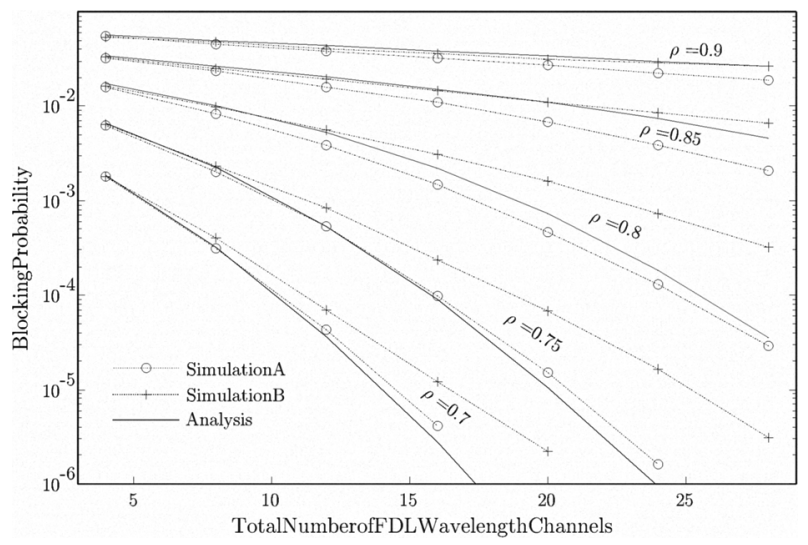

Fig. 6. Blocking Probability - Simulation and Analysis - 4 Port, 40 Output Channel Switch with two FDL Configurations (A and B)

future availability of an output channel and FDL. The burst arrival process is Poisson and burst lengths are exponentially distributed with mean length $1 \mathrm{~ms}$. The FDL base delay time is chosen as $C=3 \mathrm{~ms}$. We note that the average burst length and $C$ may be scaled together without changes to burst blocking results. Simulations were executed in batches such that the confidence interval for all points is better than $\pm 2 \%$ at a confidence level of $98 \%$. As these intervals are small, they are omitted from result plots for clarity.

Different configurations for the switch architecture presented in Fig. 1 are considered. We set the number of input/output ports, $P$, to be 4 in all cases. The number of output channels per port, $N$, is alternatively 10 or 40 . The FDL bank is configured to have alternatively multiple FDLs each carrying a single wavelength channel $(K \geq 1$ and $R=1)$, which we call "Case A", or a single FDL carrying multiple channels $(K=1$ and $1<R \leq N)$, which we call "Case B". For the same total number of channels $(L=K \cdot R)$, these two cases will generally give lower and upper bounds on blocking. Other configurations, with the same total number of $L$ channels allocated between $K$ delay lines will generally

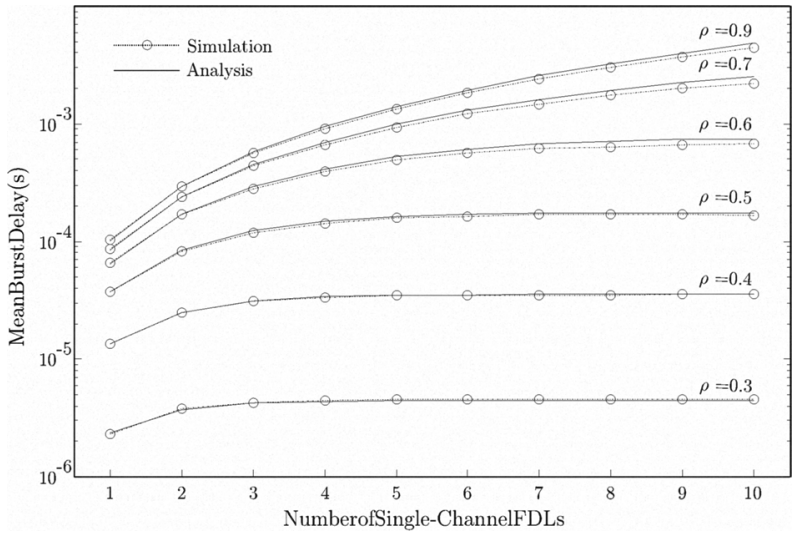

Fig. 7. Blocking Delay - Simulation and Analysis - 4 Port, 10 Output Channel Switch with Single-channel FDL Configuration (Case A)

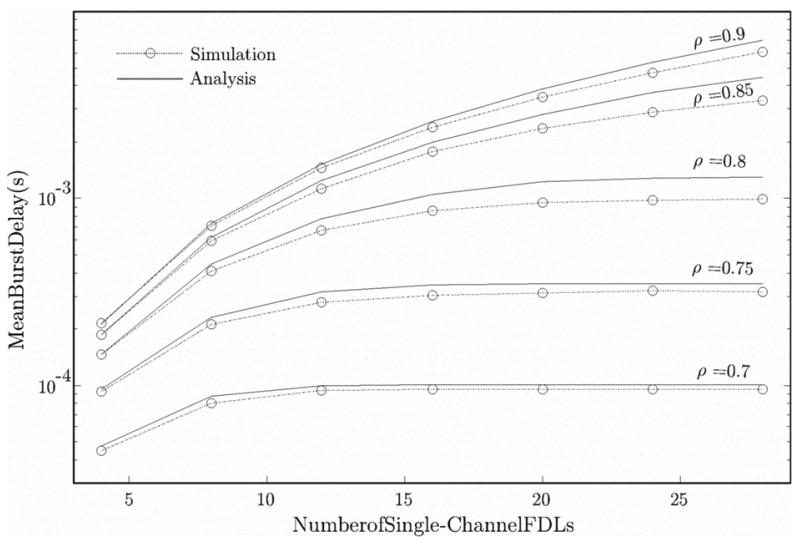

Fig. 8. Blocking Delay - Simulation and Analysis - 4 Port, 40 Output Channel Switch with Single-channel FDL Configuration (Case A)

have performances in between. (The performance improvement gained by distributing the same number of channels over larger numbers of delay lines has been previously studied in [29]).

In either Case A or B, we examine the blocking for various total numbers of channels $(L=K \cdot R)$ in the FDL bank. Results of simulation and analysis for these combinations are given in Fig. 5 and Fig. 6 for various traffic intensities per channel $\rho$. Simulation and analysis results for average burst blocking delay, for the case of single-channel FDLs (Case A), are presented in Fig. 7 and Fig. 8.

The values of blocking probability calculated by analysis for a small number of total FDL channels $(L)$ are in quite good agreement with the results obtained from simulation, for both Cases A and B, not withstanding that the analysis does not account for the difference between Cases A and B; the blocking calculation does not take account of the values of $K$ or $R$, but their product $L=K \cdot R$ only. For 10 output port channels, the error is within $\pm 10 \%$ for $L \leq 3$ and within $\pm 40 \%$ for $L \leq 5$. For 40 output channels, the error is with $\pm 10 \%$ for $L \leq 4$ and within $\pm 40 \%$ for $L \leq 12$. 
For larger numbers of FDL channels, or larger numbers of output channels, the results provide an approximate lower bound on blocking probability, with the analysis being most accurate for the case of single-channel FDLs, as the difference between performance in Cases A and B grows. Results for mean burst delay for Case A, with 10 and 40 output channels, are presented in Fig. 7 and Fig 8. respectively. Delay results follow similar patterns of error as the blocking results.

\section{CONCLUSION}

We have developed a relatively simple approximate model for an OBS node with full wavelength conversion and a shared bank of FDLs, by a novel application of ERT by way of allowing a feedback path between groups of overflowing channels. Accuracy of the model is good when the FDL bank has quite limited capacity in comparison to the capacity of the output ports, and where the FDL delays are longer than the average burst length. Future work will consider adjustments to the current model to account for traffic correlations when the FDL bank has a larger capacity. Our overall aim is to produce a model of good numerical efficiency, that may be extended to modelling and dimensioning of large networks of OBS switches. An advantage of an ERT approach is that it may approximate non-Poisson offered traffic, as normally exists at nodes in a network, because the input flow can be easily characterised by two moments. For network models, a possible limitation of the present model is that it allows only peaked offered traffic $(Z>1)$ to be modelled. In a network, the input traffic type is determined by the burst aggregation method assumed. Not to restrict the model to certain aggregation methods, we ideally require the node model to handle both peaked and smooth traffic. Such methods exist in ERT, for example by way of the Bernoulli-PoissonPascal (BPP) process [27]. This will be a topic for future work.

\section{REFERENCES}

[1] S. Yao, B. Mukherjee, S. Dixit, "Advances in Photonic Packet Switching: an Overview," IEEE Communications Magazine, vol. 38, n. 2, February 2000.

[2] T. Battestilli, H. Perros, "An introduction to optical burst switching," IEEE Communications Magazine, vol. 41, no. 8, pp. S10-S15, August 2003.

[3] P. J. Jue, V. M. Vokkarane, Optical Burst Switched Networks, Springer, 2005.

[4] P. Du, S. Abe, "Sliding Window-based Burst Assembly Method in Optical Burst Switching Networks," in Proc. 14th IEEE International Conference on Networks, vol. 2, pp. 1-6, September 2006.

[5] V. M. Vokkarane, P. J. Jue, "Prioritized burst segmentation and composite burst-assembly techniques for QoS support in optical burst-switched networks," IEEE Journal on Selected Areas in Communications, vol. 21, no. 7, pp. 1198-1209, September 2003.

[6] Y. Xiong, M. Vandenhoute, H. C. Cankaya, "Control architecture in optical burstswitched WDM networks," IEEE Journal on Selected Areas in Communications, vol. 18, no. 10, pp. 1838-1851, October 2000.

[7] C. Qiao, M. Yoo, "Optical burst switching (OBS) - a new paradigm for an optical Internet," Journal of High Speed Networks, vol. 8, no. 1, pp. 69-84, January 1999.

[8] J. Y. Wei, R. I. McFarland Jr., "Just-in-time signaling for WDM optical burst switching networks," IEEE/OSA Journal of Lightwave Technology, vol. 18, no. 12, pp. 2019- 2037, December 2000.
[9] W. Liao, C. Loi, "Providing service differentiation for optical-burstswitched networks," IEEE/OSA Journal of Lightwave Technology, vol. 22, no. 7, pp. 1651-1660, July 2004.

[10] J. Comellas, D. Cerdan, J. Conesa, S. Spadaro, G. Junyent, "Contention Resolution using Preventive Reservation in Optical Burst Switching Networks," in Proc. 2006 International Conference on Transparent Optical Networks, vol. 3, pp. 62-65, June 2006.

[11] B. Wang, N. Lella, "Dynamic contention resolution in optical burst switched networks with partial wavelength conversion and fiber delay lines," in Proc. 2004 IEEE Global Telecommunications Conference, GLOBECOM 04, vol. 3, pp. 1862-1866, December 2004.

[12] S. Lee, K. Sriram, H. Kim, J. Song, "Contention-Based Limited Deflection Routing Protocol in Optical Burst-Switched Networks," IEEE Journal on Selected Areas in Communications, vol. 23, no. 8, pp. 15961611, August 2005.

[13] B. Klusek, J. Murphy, L. P. Barry, "Cost-based burst dropping strategy in Optical Burst Switching networks," in Proc. International Conference on Transparent Optical Networks (ICTON), Paper Tu.B1.3, July 2005.

[14] F. Masetti, D. Zriny, D. Verchre, J. Blanton, T. Kim, J. Talley, D. Chiaroni, A. Jourdan, J. C. Jacquinot, C. Coeurjolly, P. Poignant, M. Renaud, G. Eilenberger, S. Bunse, W. Latenschleager, J. Wolde, and U. Bilgak, "Design and implementation of a multi-terabit optical burst/packet router prototype," in Proc. Optical Fiber Communication Conference (OFC 2002), vol. 70 of OSA Trends in Optics and Photonics Series, Optical Society of America, 2002.

[15] I. Baldine, A. Bragg, G. Evans, M. Pratt, M. Singhai, D. Stevenson, R. Uppalli, "JumpStart deployments in ultra-high-performance optical networking testbeds," IEEE Communications Magazine, vol. 43, no. 11, pp. S18-S25, November 2005.

[16] J. White, Modelling and Dimensioning of Optical Burst Switched Networks, Ph.D. Dissertation, Department of Electrical and Electronic Engineering, University of Mel- bourne, May 2007.

[17] Z. Rosberg, A. Zalesky, H. L. Vu, M. Zukerman, "Analysis of OBS Networks With Limited Wavelength Conversion," IEEE/ACM Transactions on Networking, vol. 14, no. 5, pp. 1118-1127, October 2006.

[18] G. I. Papadimitriou, C. Papazoglou, A. S. Pomportsis, Optical Switching, Wiley-Interscience, 2007.

[19] X. Lu, B. L. Mark, "Performance Modeling of Optical-Burst Swirching With Fiber Delay Lines," IEEE Transactions on Communications, vol. 52, n. 12, December 2004.

[20] M. Yoo, C. Qiao, S. Dixit, "QoS Performance of Optical Burst Switching in IP-Over-WDM Networks," IEEE Journal on Selected Areas in Communications, vol. 18, n. 10, October 2000.

[21] P. Fan, C. Feng, Y. Wang, N. Ge, "Investigation of the Time-OffsetBased QoS Support with Optical Burst Switching in WDM Networks," IEEE International Conference on Communications, 2002.

[22] F. Callegati, "Optical Buffers for Variable Length Packets," IEEE Communications Letters, vol. 4, n. 9, September 2000.

[23] W. Rogiest, D. Fiems, K. Laevens, and H. Bruneel, "Tracing an optical buffer's performance: An effective approach," Lecture Notes in Computer Science, NET-COOP 2007 Special Issue, 4465:185-194, 2007.

[24] W. Rogiest, D. Fiems, K. Laevens, H. Bruneel, "Exact Performance Analysis of FDL Buffers with Correlated Arrivals," IFIP International Conference on Wireless and Optical Communications Networks, 2007.

[25] C. Gauger, "Contention Resolution in Optical Burst Switching Networks," COST 266, Technical Committee Telecommunications.

[26] P. Reviriego, A. M. Guidotti, C. Raffaelli, J. Aracil, "Blocking models of optical burst switches with shared wavelength converters: exact formulations and analytical approximations," Photonic Network Communications, vol. 16, n. 1, 2008.

[27] A. Girard, Routing and Dimensioning in Circuit-Switched Networks, Addison-Wesley Longman Publishing Company, 1990.

[28] ITU-D, Study Group 2, Teletraffic Engineering Handbook, URL:http://www.itu.int/ITU-D, last visited March 2009.

[29] C. M. Gauger, H. Buchta, E Patzak, "Integrated Evaluation of Performance and TechnologyThroughput of Optical Burst Switching Nodes Under Dynamic Traffic," Journal of Lightwave Technology, vol. 26, n. 13, pp. 1969-1979, July 2008.

[30] L. Kleinrock, Queueing Systems Volume I:Theory, John Wiley \& Sons Inc, 1975.

[31] O. Hudousek, "Evaluation of the Erlang-B formula," in Proc. RTT 2003, pp. 80-83, Bratislava: FEI, Slovak University of Technology, 2003.

[32] Opnet Technologies, Opnet Modeler, URL:http://www.opnet.com/ solutions/network_rd/modeler.html, last visited July 2009. 